\title{
Novel WDM Access Network Featuring Self-healing Capability and Flexible Extensibility
}

\author{
Chen Feng, Chaoqin Gan, Ziyue Gao and Chenwei Wu \\ Key Laboratory of Specialty Fiber Optics and Optical Access Networks, School of Communication \\ and Information Engineering, Shanghai University, No. 149, Yanchang Road, Zhabei District, \\ 200072 Shanghai, China \\ Chaoqin Gan: cqgan@shu.edu.cn
}

Keywords: wavelength division multiplexing access network; ring topology; flexible extensibility; high reliability

\begin{abstract}
A novel WDM access network is proposed in this paper. By designing the remote node and the extended node, the network has the flexible extensibility. By using ring topology, the architecture of network is constructed. It makes the network not only have the high reliability, but also can cover large access areas. By simulation, the mentioned functions are demonstrated. The simulating results show the network has good performance.
\end{abstract}

\section{Introduction}

With the exponential growth of the internet demand and high bandwidth applications, higher requirements such as larger capacity and more flexible extensibility are raised to optical access network[1]-[3]. To meet such requirements, ring-based network is considered as a good candidate solution. Owing to similar structure, ring-based network can consolidate metro and access resources into a single topology and provide larger capacity easily [4,5]. On the other hand, as the rapid growth of data traffic on network, reliability receives more and more attention. And relying on the superiority of its physical topology, ring-based network can provide fiber-fault protection easily [6]. Both dual-fiber and single-fiber ring-based schemes have been proposed to realize higher reliability [7]-[9].

In this paper, we propose a novel ring-based WDM access network not only can support fiber-fault protection, but also provide extensibility and high capacity.

\section{Principle of Operation}

The ring-based WDM access network is shown in Fig. 1. In main ring, this network contains one optical line terminal (OLT) and m remote nodes (RNs). Each RN is shared by n optical network units (ONUs), so entire network can support $\mathrm{m} * \mathrm{n}$ ONUs. The main ring is divided into upper and lower branch by $\mathrm{RN}_{\mathrm{m} / 2}$ and $\mathrm{RN}_{\mathrm{m} / 2+1}$. In upper branch, downstream signals are transmitted clockwise, and upstream signals are transmitted anticlockwise. As opposed to upper branch, downstream and upstream signals are transmitted in anticlockwise and clockwise direction respectively in lower branch. By using extended nodes (ENs), main ring can be extended. The extended topologies can include ring, bus, tree, and so on to meet the new requirements of current network.

The structure of OLT and ONU is shown in Fig. 2. In OLT, there are $m * n$ transmitters, one erbium-doped fiber amplifiers (EDFAs), two circulators (Cir), one wavelength selective switch (WSS), one Arrayed Waveguide Grating (AWG), one receiver array and one monitor. The WSS works as the wavelength router in the system. It decides the output port for the downstream signals. Each ONU contains one Cir, one $1 \times 2$ optical splitter, receiver, wavelength converter (WC), and one reflective semiconductor optical amplifier (RSOA).

The structure of RN is illustrated in Fig. 3. Each RN contains two dense wavelength division multiplexers (DWDMs), two couplers, two Cirs, one $2 * \mathrm{~N}$ AWG, one wavelength block (WB) and two extended coarse wavelength division multiplexers (CWDMs). 


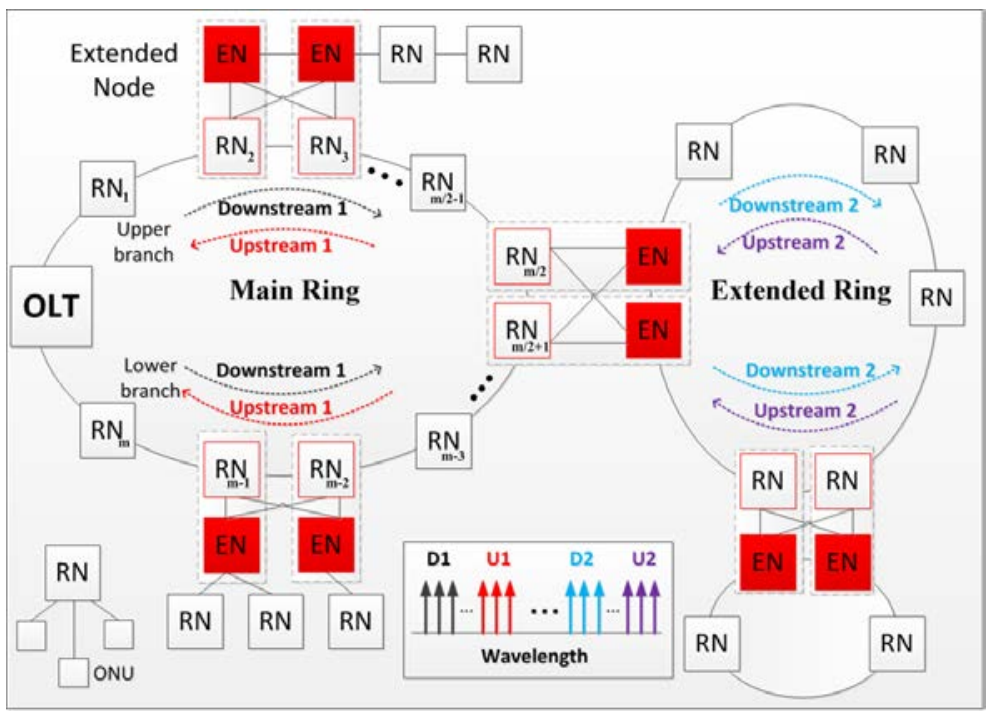

Fig.1. Schematic of network.

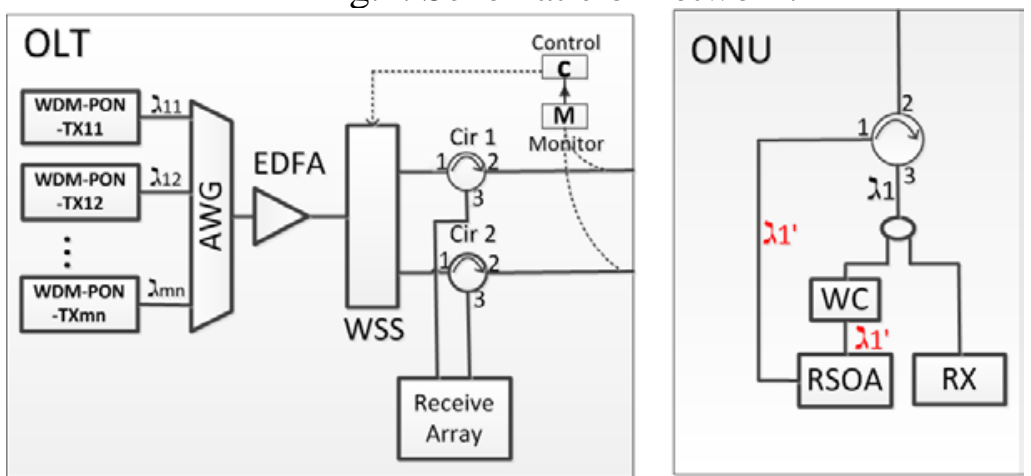

Fig.2. Proposed structures of OLT and ONU.

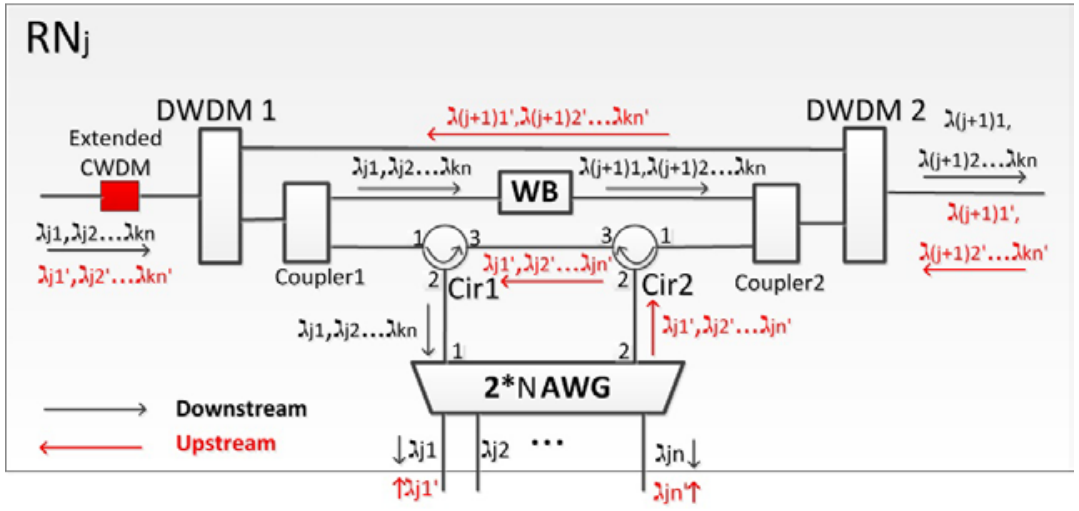

Fig.3. Schematic of the RN's configuration.

In normal mode, $\mathrm{m} * \mathrm{n}$ transmitters in OLT working on wavelengths $\lambda_{11} \lambda_{12} \cdots \lambda_{m n}$ are used to transmit the light source of downstream signals to each ONU. Here, the wavelengths are identified by two indexed. The $\lambda_{i j}$ represents the wavelength transmitted to the $\mathrm{ONU}_{\mathrm{j}}$ connecting with $\mathrm{RN}_{\mathrm{i}}$. After multiplexed by AWG, these downstream signals are divided into two parts by WSS. Here, WSS works as the wavelength router in the network to control the direction of all the downstream signals. In normal mode, downstream signals $\lambda_{11}-\lambda_{m / 2 n}$ are routed to $\mathrm{RN}_{1}$ by $\mathrm{Cir}_{1}$ in upper branch, while downstream signals $\lambda_{(m+2) / 21}-\lambda_{m n}$ are routed to $\mathrm{RN}_{\mathrm{m}}$ by $\mathrm{Cir}_{2}$ in lower branch. The $\mathrm{M}$ (monitor) constantly detects the optical signals from upper branch and the lower branch of the network. These signals transmitted in two branches are operated in mirror method. Taking $\mathrm{RN}_{\mathrm{j}}$ in upper branch as an example, downstream signals $\lambda_{j 1} \lambda_{j 2} \cdots \lambda_{k n}$ ( $\mathrm{k}$ is the last RN of upper branch in the main ring) coming from $\mathrm{RN}_{\mathrm{j}-1}$ are divided into two parts by coupler ${ }_{1}$ after passing through $\mathrm{DWDM}_{1}$. One is sent into WB. And signals $\lambda_{j 1} \lambda_{j 2} \cdots \lambda_{j n}$ are blocked via WB to avoid further wavelength crosstalk in the later 
propagation. Then Residual signals $\lambda_{(j+1) !} \lambda_{(j+1) 2} \cdots \lambda_{k n}$ are transmitted to next $\mathrm{RN}_{\mathrm{j}+1}$. The other are demultiplexed by $2 * \mathrm{~N}$ AWG and sent to ONUs after passing through the $\mathrm{Cir}_{1}$. At ONU, downstream signals are divided into two parts by $1 \times 2$ optical splitter: one is demodulated by a receiver, while the other utilized to provide light source for upstream signals is injected into one RSOA and re-modulated. It should be noted that the proposed network is a bidirectional transmission network by employing RSOA in ONU, so Rayleigh backscattered noise can be a severe problem. To solve this problem, wavelength converter (WC) is used to shift the wavelength of signals to another wavelength band before downstream signals are re-modulated by RSOA. After being re-modulated, upstream signals $\lambda_{j 1}{ }^{\prime} \lambda_{j 2}{ }^{\prime} \cdots \lambda_{k n}$ ' are transmitted back to $\mathrm{RN}_{\mathrm{j}}$ over the same route. At the same time, upstream signals $\lambda_{(j+1) 1}{ }^{\prime} \lambda_{(j+1) 2}{ }^{\prime} \cdots \lambda_{k n}$ ' coming from $\mathrm{RN}_{\mathrm{j}+1}$ to $\mathrm{RN}_{\mathrm{k}}$ are separated by DWDM 2 and directly routed to $\mathrm{DWDM}_{1}$. Then signals $\lambda_{j 1}{ }^{\prime} \lambda_{j 2}{ }^{\prime} \cdots \lambda_{j n}$ ' coming from the port 2 of AWG are combined with $\lambda_{(j+1) 1}{ }^{\prime} \lambda_{(j+1) 2} ' \cdots \lambda_{k n}{ }^{\prime}$ and transmitted back to OLT.

By above working mechanism, the fiber-fault problem can be solved by appropriately setting the wavelengths for upper and lower branch RNs, respectively. In case of failure occurring in RN or fiber, the WSS in OLT will need to be adjusted appropriately. To state the principle of such adjustment, it is assumed that fiber between $\mathrm{RN}_{\mathrm{i}}$ and $\mathrm{RN}_{\mathrm{i}+1}$ fails. When the $\mathrm{M}$ in OLT detects the loss of the upstream wavelength signals, the $C$ (control) module will control the WSS to reassign the wavelengths to restore the affected traffic as far as possible. Downstream signals $\lambda_{11}-\lambda_{\text {in }}$ are routed to the upper branch of the main ring and $\lambda_{(i+1) 1}-\lambda_{m n}$ are routed to the lower branch. By that configuration, the transmission can be restored from the single failure.

The principle of extension in the network is demonstrated in Fig.4. When the migration is needed to extend network, two adjacent RNs in main ring will be chosen as the output interface for extension. By connecting with the extended CWDM in these two RNs, two additional ENs can realize flexible extension and mutual protection. According to the design, one $\mathrm{RN}$ is considered as the master $\mathrm{RN}$ as well as the corresponding EN. In the Fig.4, the $\mathrm{RN}_{\mathrm{j}}$ and the $\mathrm{EN}_{1}$ work as the master nodes. Under the normal situation, the $\mathrm{SW}_{2}$ and $\mathrm{SW}_{3}$ in $\mathrm{EN}_{1}$ are triggered to port 1(black line). At the same time, $\mathrm{SW}_{2}$ and $\mathrm{SW}_{3}$ in $\mathrm{EN}_{2}$ are triggered to port 2 (black line). In this method, the $\mathrm{EN}_{1}$ is worked as converge point of the extended ring. All the downstream and upstream signals are transmitted through $\mathrm{EN}_{1}$. The M of the SW1 keeps detecting the link connectivity between the main ring and the extended ring. When the link break occurs, the $\mathrm{C}$ in $\mathrm{EN}_{1}$ will first reconfigure the $\mathrm{SW}_{1}$ to port 1 to try to recover the network from the link failure. If this configuration still does not works, the $\mathrm{EN}_{2}$ will works as the master node of the extended ring by trigger the $\mathrm{SW}_{2}$ and $\mathrm{SW}_{3}$ in $\mathrm{EN}_{2}$ to port 1 . The WSS in two ENs are controlled by $\mathrm{C}$ and $\mathrm{M}$ in the same way as the WSS in OLT.

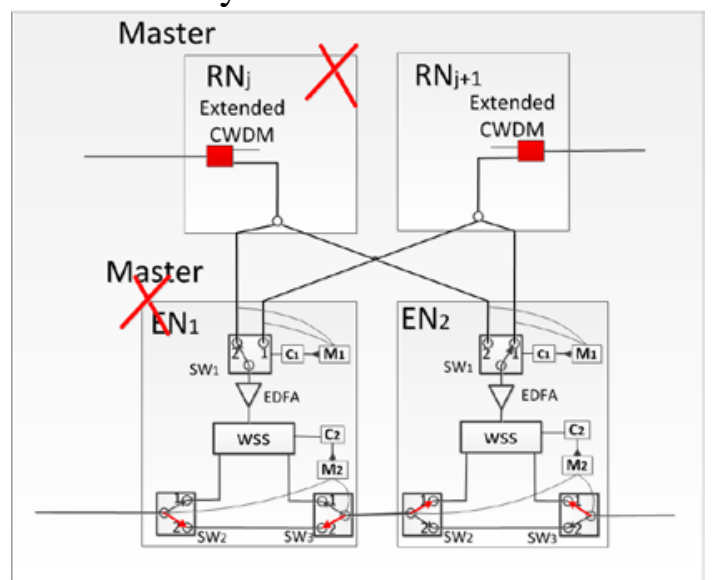

Fig.4. Schematic of the EN's configuration

In the proposed scheme, the ring-based network can be easily and flexibly extended through modularized EN. Thus both the reach distance and the number of subscribers in the network can be largely extended with the minimum migration cost. At the same time, the EN can realize the mutual 
protection in case of fiber-fault or nodes failure. Furthermore, the single ring topology itself also has the ability of self-healing, which makes the network more resilient to the concurrent failures.

\section{Analysis and Simulation}

The simulation setup of the network is depicted in Fig. 5. To demonstrate the transmission performance of the network, three DFB lasers operating at $1550 \mathrm{~nm}, 1550.8 \mathrm{~nm}$, and $1551.6 \mathrm{~nm}$ are used. These three wavelengths are used to transmit downstream signals to $\mathrm{RN}_{2}, \mathrm{RN}_{4}$ and $\mathrm{RN}_{5}$ in the extended ring, respectively. The output power of each laser is set to be $0 \mathrm{dBm}$. In addition, three wavelengths are modulated by phase modulator (PM). PM adopts non-return to zero (NRZ) driven at 10 Gbps. Then downstream signals at $1550.8 \mathrm{~nm}$ and $1551.6 \mathrm{~nm}$ are transmitted to $\mathrm{RN}_{4}$ in anticlockwise through $\mathrm{FF}_{5}$. And downstream signals at $1550 \mathrm{~nm}$ are transmitted to $\mathrm{RN}_{1}$ clockwise through $\mathrm{FF}_{1}$. The transmission length between OLT and $\mathrm{RN}_{1}\left(\mathrm{RN}_{5}\right)$ is $10 \mathrm{~km}$, and transmission length between two adjacent RN is $5 \mathrm{~km}$. The DF connecting ONU with RN is $2 \mathrm{~km}$. Both working and protection mode are simulated. Under protection mode, fiber-fault occurs in $\mathrm{FF}_{5}$ between OLT and $\mathrm{RN}_{4}$.

The measured BER curves for downstream signals operating at 10 Gbps data rate are plotted in Fig.6. It shows that, in normal mode, downstream signals at $1550.8 \mathrm{~nm}$ and $1551.6 \mathrm{~nm}$ have almost the same minimum received power at a BER of $10^{-9}$ (around $-21.5 \mathrm{dBm}$ ). And to downstream signals at $1550 \mathrm{~nm}$, there is nearly $0.9 \mathrm{dBm}$ power penalty caused by extra $5 \mathrm{~km}$ fiber transmission. Under protection mode, there are big changes in transmission performances of downstream signals at 1550.8 $\mathrm{nm}$ and $1551.6 \mathrm{~nm}$. And power penalty of two signals caused by the additional transmission distance is $2 \mathrm{dBm}$ and $2.4 \mathrm{dBm}$ under protection mode. However due to the transmission distance unchanged, the minimum received powers of $1550 \mathrm{~nm}$ downstream signals at a BER of $10^{-9}$ are the same about $-20.5 \mathrm{dBm}$.

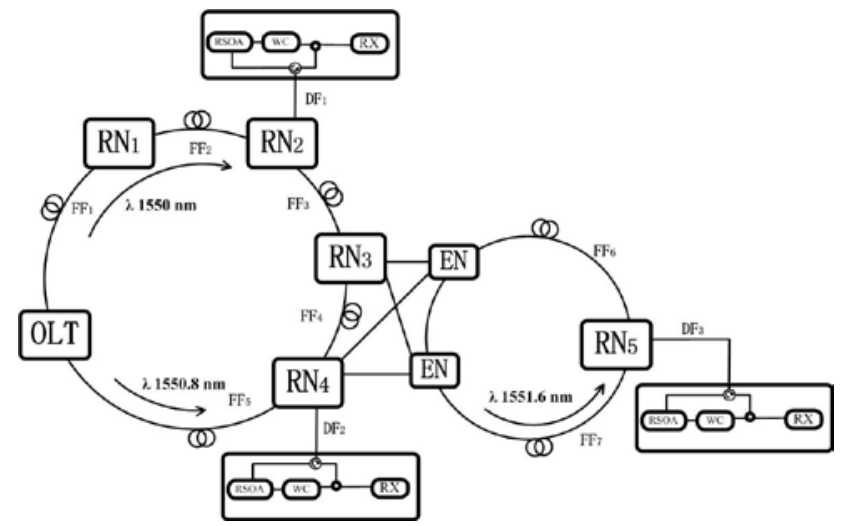

Fig.5. Simulation setup of proposed WDM access network.

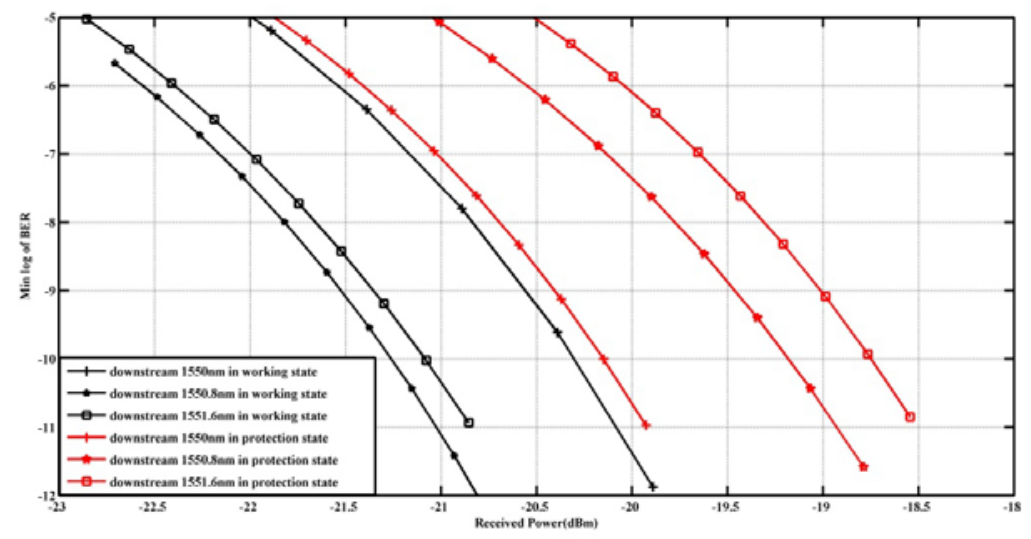

Fig.6. BER curves of the downstream signals.

The measured BER curves for upstream signals operating at 2.5 Gbps data rate are plotted in Fig.7. Due to the different transmission distance, there is nearly $1 \mathrm{dBm}$ power penalty among downstream 
signals at $1550 \mathrm{~nm}, 1550.8 \mathrm{~nm}$ and $1551.6 \mathrm{~nm}$ (around $-23.2 \mathrm{dBm},-24.8 \mathrm{dBm}$, and $-24.2 \mathrm{dBm}$ ) in working mode. Like the simulation result of downstream signals, under protection mode, there are still big changes in transmission performances of upstream signals at $1550.8 \mathrm{~nm}$ and $1551.6 \mathrm{~nm}$. And power penalty caused by the additional transmission distance is $2.8 \mathrm{dBm}$ and $2.3 \mathrm{dBm}$, respectively. Similar to the case of downstream signals, due to the transmission distance unchanged, the minimum received powers of upstream signals at 1550 at a BER of $10^{-9}$ remains the same.

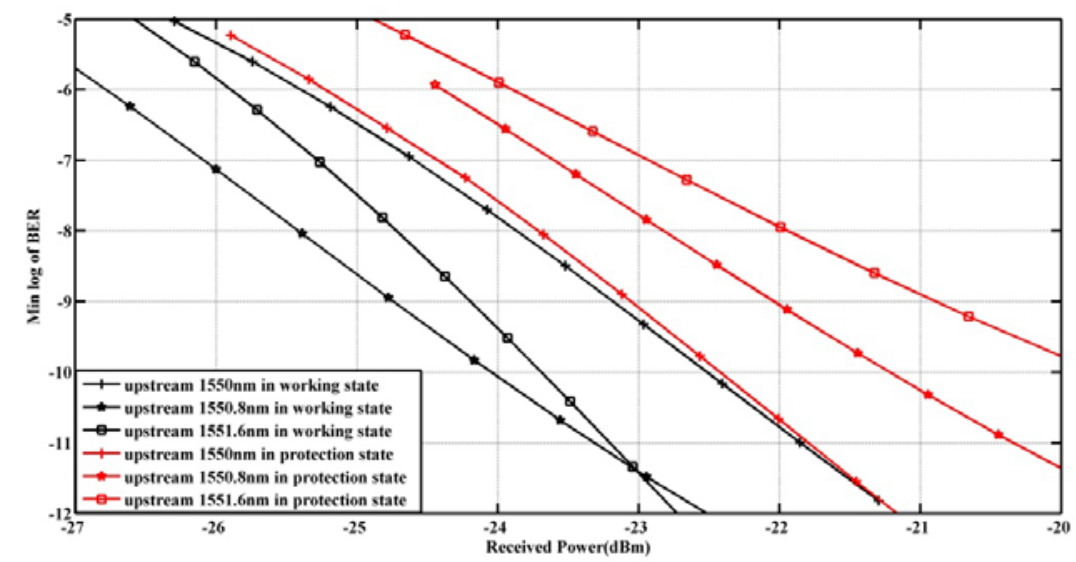

Fig.7. BER curves of the upstream signals.

\section{Conclusion}

We propose and demonstrate a ring-based WDM access network. The EN and RN is designed to make the network have the extensibility. It can flexibly configure and adjust scale of network. Through the extended CWDM and corresponding ENs, the origin network can be conveniently and modularized extended to the dual-ring architecture enlarging the scale of network. Thus both the reach distance and the number of subscribers in the network can be largely extended with the minimum migration cost. In addition, by using ring-based topology, the network has protection mode. The affected traffic can be restored against fiber faults occurring in both the feeder fiber and the extended nodes by using the proposed protection mode. These make the network have high reliability. All of the above indicate the network has great resilience. Finally, we realize and demonstrate the mentioned functions of the network by the simulation. Simulation results show the network has good transmission performance of downstream and upstream signals.

\section{Acknowledgment}

This work is supported by Programs of Natural Science Foundation of China (No.61132004, 61275073 and 61420106011), Shanghai Science and Technology Development Funds (No. 13JC1402600 and 14511100100), Shanghai Leading Academic Discipline Project (No.S30108), Shanghai Economics and Information Project(No.CXY-2013-93) and Key Laboratory of Specialty Fiber Optics and Optical Access Networks, Shanghai University (No.SKLSFO2012-05).

\section{Reference}

[1] Lee, C.H., Sorin,W.V.,Kim, B.Y. Fiber to the home using a PON infrastructure. J. Lightwave Technology.2006, 24(12), pp:4568-4583.

[2] Park, S.J., Lee, C.H., Jeong, K.T., Park, H.J., Ahn, J.G., Song, K.H., Fiber-to-the-home services based on wavelength-division-multiplexing passive optical network, Journal ofLightwave Technology, 2004, 22(11), pp:2582-2591. 
[3] Saliou, F., Chanclou, P., Laurent, F., Genay, N., Lazaro, J.A., Bonada, F. andPrat, J. Reach Extension Strategies for PassiveOptical Networks, Optical Communications and Networking, 2009, 1(4):C51-C60.

[4] W.-T. Shaw, G. Kalogerakis, S.-W. Wong, Y.-L. Hsueh, N.Cheng, S.-H. Yen, M. E. Marhic, and L. G. Kazovsky, "MARIN: metro-access ring integrated network", IEEE Global Telecommunications Conf., 2006. GLOBECOM ’06, San Francisco,CA, Nov. 27-Dec. 1, 2006, pp. 1-5.

[5] Chen Chen, Chongfu Zhang, Qiongli Zhang, Kun Qiu, “A Scalable Metro-Access Integrated Network System with Reconfigurable WDM Central Ring and High-quality OFDMA Access Trees”, ACP, Guangzhou, pp.1-3, 2012.

[6] C. H. Yeh, C. W. Chow, Y. F. Wu, F. Y. Shih and S. Chi, "Self-protected time-division-multiplexed passive access networks in tree and ring topology architectures", Photonic Network Communications, vol. 23, no. 2 , pp. 130-136, 2012.

[7] Esmail, M.A., Fathallah, H., Fiber Fault Management and Protection Solution for Ring-and-Spur WDM/TDM Long-Reach PON, Global Telecommunications Conference (GLOBECOM 2011), 2011, pp: 1-5.

[8] Yeh, C.H., Chow, C.W.,Signal Re-modulation Ring WDM Passive Optical Network with Rayleigh Backscattering Interferometric Noise Mitigation, Communications Letters, 2011, 15(10), pp: 1114-1116.

[9] Chen Feng, Chaoqin Gan, Yan Gong and Chenwei Wu, "High-reliable WDM Access Network Based on Alternate Transmission Mode Featuring Power Optimization and Network Stability”, Optical Switching and Networking, vol. 18, pp. 11-19, 2015. 